\title{
A propos de la lutte contre le typhus exanthématique et la fièvre récurrente épidémiques
}

\author{
Mooser, $\mathrm{H}$
}

Abstract: Ces deux maladies sont une des conséquences de la guerre et de la misère. Prophylaxie, et lutte contre elles, semblent être, théoriquement, d'une facilité élémentaire

DOI: https://doi.org/10.1017/s1026881200011090

Posted at the Zurich Open Repository and Archive, University of Zurich ZORA URL: https://doi.org/10.5167/uzh-154932

Journal Article

Published Version

Originally published at:

Mooser, H (1942). A propos de la lutte contre le typhus exanthématique et la fièvre récurrente épidémiques. International Review of the Red Cross, 24(278):113-120.

DOI: https://doi.org/10.1017/s1026881200011090 


\section{H. MOOSER,}

professeur à la Faculté de médecine et divecteur de l'Institut d'hygiène de l'Université de Zurich

\section{A propos de la lutte contre le typhus exanthématique et la fièvre récurrente épidémiques ${ }^{1}$}

Ces deux maladies sont une des conséquences de la guerre et de la misère. Prophylaxie, et lutte contre elles, semblent être, théoriquement, d'une facilité élémentaire ${ }^{2}$.

La source de contagion se trouve chez l'homme infecté ${ }^{3}$. Les poux de l'homme, et surtout les poux des vêtements, sont les seuls agents transmetteurs. Tout le cycle évolutif du pou, en partant de l'œuf jusqu'au stade qui permet la propagation, se développe sur l'homme. Il convient donc de nettoyer à fond le corps, l'habillement et la literie. Les habits et la literie peuvent être désinfestés par :

I) des procédés mécaniques ;

2) des procédés chimiques ;

3) des procédés physiques.

\section{Procédés mécaniques.}

Il s'agit là de moyens de fortune qui ne suffiront jamais à eux seuls car il importe d'envisager le nettoyage à fond des

${ }^{1}$ Les lecteurs trouveront également des indications sur la lutte contre le typhus exanthématique dans le tome XXXIII, nos 3-4 (mars-avril I94I) du Bulletin mensuel de l'Office international d'hygiène publique, Paris, boulevard Saint-Germain, I95.

2 Cf. Circulaire du Service fédéral de I'hygiène publique, aux autorités sanitaires cantonales, concernant les mesures à prendre contre le typhus exanthématique (27 janvier 1942). Voir ci-après pp. 135-138 (N.d.l.R.),

${ }^{3}$ La Revue internationale de la Croix-Rouge a publié, $\mathrm{n}^{\circ}$ de janvier $193^{8}$, pp. 35-62, avec hors-texte, le document suivant: " Rapport final sut l'épouillage des troupes dans la zone de l'avant", que son auteur, M. le colonel J. Thomann, pharmacien-chef de l'armée suisse, avait présenté à la XII ession de la Commission internationale permanente d'études du matériel sanitaire.

Cf. dans le même no de la Revue internationale, pp. 63-67, l'étude de MM. C. Iliesco, médecin inspecteur général (Roumanie), et C. Suhateanu : "Sur le typhus exanthématique en Roumanie ", et, février 1936, pp. 97-I05, le rapport de M. le $D^{\mathbf{r}}$ E. Moguilewitch (U.R.S.S.), chef de l'Institut sanitaire scientifique et expérimental de l'armée rouge : "La lutte contre les poux dans les armées en campagne". (N.d.l.R.). 


\section{H. Mooser}

vêtements; de plus, les cheveux ainsi que les poils pubiens du porteur de poux doivent être rasés.

\section{Procédés chimiques.}

Ils comprennent l'application de substances toxiques sous forme de gaz ou de liquides, destinées à tuer les poux et leurs œufs, par exemple :

\section{a) acide cyanhydrique.}

C'est l'agent le plus actif dans la lutte contre tous les insectes. Son utilisation cependant, doit être réservée pour le temps de paix et se faire sous la direction d'un personnel particulièrement spécialisé. L'improvisation de son emploi, lors d'une épidémie, entraînerait trop de risques.

b) gaz sulfureux $\left(\mathrm{SO}_{2}\right)$ obtenu par la combustion de briquettes de soufre, ou émané de bouteilles qui le contiennent à l'état comprimé.

A l'aide de gaz sulfureux on peut désinfecter une chambre, par exemple un dortoir avec les habits et la literie qui s'y trouvent sans courir le danger d'une intoxication. Tous les objets infestés, habits, literie, couverturres, etc., seront bien étalés et suspendus à des ficelles ou à des fils de fer fixés dans le local, de telle sorte que les vapeurs sulfureuses puissent pénétrer partout. Cette méthode peut être improvisée partout où l'on dispose de chambres pouvant être fermées hermétiquement. Elle ne nécessite aucun appareil coûteux ni compliqué.

On emploie alors du soufre et du salpêtre (nitrate de potasse) que l'on arrose avec un peu d'alcool, pour faciliter la combustion. Pour éviter tout danger d'incendie, on brûle ce soufre dans une grande cuvette ou un seau métallique.

La désinfection dure de 3 à 4 heures. Par $\mathrm{m}^{3}$ de capacité de la chambre, il faut brûler environ 60 gr. de soufre.

c) Solutions désinfectantes.

Pour tuer les poux se trouvant dans les habits, on peut également utiliser : 


\section{Typhus exanthématique et fièvre récurrente épidémiques}

a) de l'acide phénique, aux concentrations suivantes:

$5 \%$ appliqué pendant 40 minutes ;

$3 \%$ appliqué pendant 3 heures ;

I\% appliqué pendant 4 heures;

b) du savon au crésol à 3-5\%, appliqué pendant $\mathrm{I}$ heure ; du savon au crésol à $I \%$, appliqué pendant 4 heures.

Les habits et la literie doivent être trempés, pièce par pièce, dans la solution, pour que le liquide désinfectant pénètre chacune d'elles. En utilisant à chaud l'acide phénique ou les solutions de savon au crésol, les poux et les lentes succombent plus rapidement.

Cette méthode d'épouillage d'une petite quantité d'habits ne peut se pratiquer que dans les demeures privées et dans les petits hôpitaux ; elle enrayera avec certitude l'infection par les excréments de poux qui se trouvent dans les vêtements.

De plus, parmi les moyens efficaces pour tuer les poux, it faut citer également le pétrole, la benzine, le benzol et le kérosène.

\section{Procédés physiques - la chaleur.}

Les poux et les lentes succombent d'une façon certaine sous l'influence de la chaleur, sèche ou humide. Et l'utilisation decet agent physique est très avantageuse, car il agit beaucoup plus rapidement que les vapeurs sulfureuses.

La chaleur sèche à $80^{\circ}$, tue les poux et les lentes en $15 \mathrm{mi}$ nutes; à $60^{\circ}$, elle les détruit à coup sûr en $I$ heure.

La vapeur d'eau, à partir de $60^{\circ}$ tue les poux et les lentes en quelques minutes. Cependant l'action de la chaleur devrait durer au moins I heure pour obtenir un résultat certain, c'est à dire la garantie que les vapeurs ou l'air chaud aient pénétré dans toutes les parties de l'habit. Le fer à repasser chaud, appliqué sur les vêtements humectés, est aussi un excellent moyen d'épouillage. Faute de mieux on les trempera dans de l'eau bouillante.

Nettoyage et épouillage du corps.

Pour obtenir une action efficace, il faut raser le corps et. le nettoyer ensuite avec du savon noir et de l'eau chaude. 


\section{H. Mooser}

Toutefois, au lieu de raser les poils infestés, des régions pubienne et anale, on peut faire usage de frictions à la pommade grise ou au calomel.

Les moyens suivants pour la destruction des poux et des lentes dans les parties poilues du corps sont plus spécialement recommandés :

I. mélange de 3 parties de paraffine avec 7 parties d'huile d'olive, de sésame, ou d'une autre huile;

2. frictions énergiques avec du pétrole ou du kérosène;

3. récemment, les auteurs anglais ont prescrit l'emploi de l'huile lourde de goudron de houille comme moyen très efficace contre les poux et les lentes. Les poux sont immédiatement tués à son contact. Quant aux vapeurs de cette huile, elles les tueraient en une heure. Avec ces vapeurs, on peut également désinfester les habits et la literie. Cependant, étant donné que l'huile de goudron est inflammable, la vaporisation doit se faire avec précautions sur une flamme protégée et dans des locaux hermétiquement fermés, comme dans le cas des applications de vapeurs de.soufre.

Ce ne sont pas seulement les femmes qui s'opposent à la taille des cheveux, mais aussi les hommes, et cela spécialement pendant les périodes de froid. Dans ces circonstances particulières, la destruction des poux de la tête et de leurs lentes peut être facilement réalisée par l'emploi, sous forme de frictions, de pétrole ou de kérosène ( 75 parties de pétrole pour 25 parties d'huile de lin ou de sésame). Lors de cette opération il est indispensable de se protéger les yeux. Le chloroforme et l'éther appliqués sur les cheveux ont également une action efficace.

\section{Conseils pratiques.}

Dans les pays peu civilisés, et en temps d'épidémie, ce n'est guère que l'improvisation sur place qui entre en ligne de compte. Les installations de désinfection, telles que les autoclaves ou les wagons-douches utilisés dans les armées, ne sont pas disponibles. Néanmoins, dans les villes et les grandes agglomérations, il est relativement facile d'improviser une installation, car on $y$ trouve toujours des fabriques et des locomotives pouvant fournir 


\section{Typhus exanthématique et fièvre récurrente épidémiques}

de l'air chaud ou de la vapeur surchauffée, ainsi que de l'eau chaude pour le nettoyage du corps. Des fours et des installations de torréfaction peuvent aussi être utilisés à cet effet. Dans les villes, on dispose en outre, presque toujours, des locaux nécessaires. En revanche, l'épouillage dans les régions campagnardes s'avère, en règle générale, extrêmement difficile. D'autre part, il faut toujours tenir compte du fait que l'épouillage ne devient un moyen de lutte efficace contre le typhus exanthématique et la fièvre récurrente épidémiques que lorsqu'un nombre éievé de personnes, des centaines d'individus à la fois, subissent cette désinsectisation. En outre, ceux qui viennent d'être désinfectés ne doivent pas entrer en contact avec ceux qui sont encore infestés. L'idéal serait de pouvoir épouiller en un seul jour un camp de réfugiés, un camp de quarantaine ou un village entier, ce qui n'est pratiquement pas réalisable. Cela demanderait une vaste organisation et des moyens considérables. De plus, il faut encore penser au ravitaillement des individus, si la désinfection se prolonge. En général, il ne faut pas compter sur la collaboration de la population, car celle-ci ne comprend pas que les poux ont quelque chose à faire avec la maladie. Cependant, il n'est pas tout à fait inutile d'instruire la population sur le danger que présentent les poux, soit par des affiches, des conférences ou des articles dans les journaux.

Pour l'épouillage d'une population paysanne et de fugitifs, dans la Chine du Nord-Ouest, par exemple, les membres de la Commission de la Société des Nations improvisèrent, en 1938, des armoires en bois démontables, faciles à transporter, reliées par un tuyau en fer à un fourneau de fonte. A l'aide d'une pompe à air, employée partout en Chine, ils obtinrent dans ces armoires une température de $79^{\circ}-90^{\circ}$ en l'espace de $20-30$ minutes, en faisant passer de l'air sur du charbon incandescent.

Pendant ce temps on faisait chauffer des fers à repasser sur le fourneau pour les appliquer ensuite sur les vêtements humectés des porteurs de poux.

Le succès de l'improvisation dépend donc de l'esprit inventif du personnel de désinfection. On ne peut établir de règles rigides pour l'épouillage. Selon les possibilités, on emploiera le soufre, la chaleur, sèche ou humide. Quant aux équipes de désinfection 


\section{H. Mooser}

elles disposeront d'un ingénieur ou d'un technicien habile et d'ouvriers capables. Ceux-ci seront en général recrutés sur place, tandis que le chef et le personnel technique (ingénieur, techniciens) devront appartenir à l'état-major permanent d'une mission ad hoc.

L'épouillage des individus sains n'est qu'une partie du problème de la lutte contre la fièvre récurrente épidémique et le typhus exanthématique; les malades doivent être strictement isolés, et leurs vêtements soigneusement désinfectés. Or, comme ces deux maladies surviennent surtout lors des saisons froides, pendant que les habits sont à la désinfection, leurs propriétaires doivent attendre, parfois plusieurs heures, avant de pouvoir les remettre, surtout lorsque l'épouillage est pratiqué à l'aide de gaz sulfureux. Cette attente n'est donc possible que si l'on a prévu, soit des locaux séparés et chauffés, pour hommes et femmes, soit des vêtements de rechange propres, ou, au moins, des couvertures de laine.

Ainsi, seul, le gaz sulfureux et la chaleur sèche entrent en ligne de compte en hiver. Et pourtant, dans cette saison, on ne peut pas licencier les épouillés de l'établissement de désinfection vêtus de vêtements humides.

En hiver, la population pauvre de l'Orient porte sur elle tout ce qu'elle possède comme vêtements. C'est pourquoi on devrait épouiller le tout à la fois; pendant ce temps les personnes devant être épouillées doivent être baignées, rasées, etc., et provisoirement revêtues de vêtements propres, s'il n'y a pas de locaux chauffables disponibles pendant les grands froids. Le mieux serait que les vêtements fussent toujours épouillés au moment où l'épouillage et le nettoyage du corps ont pris fin. Cette circonstance ne peut pas toujours être obtenue, surtout quand on n'a pas une installation d'air chaud à sa disposition et qu'il faut épouiller au gaz sulfureux. Si l'on ne dispose pas de locaux chauffables ni de couvertures de laine propres pour protéger les individus contre le froid, l'épouillage portera tout d'abord sur une partie des vêtements. Après l'avoir achevé, on procédera à l'épouillage des sous-vêtements et pendant ce temps à l'épouillage des personnes. En attendant la fin de 


\section{Typhus exanthématique et fièvre récurrente épidémiques}

ces opérations, les épouillés remettront les habits déjà désinfectés.

Dans les villes où de grands locaux chauffables sont à disposition, ce qui ne sera pas toujours le cas vu le manque de combustibles, il n'est pas nécessaire de mettre des vêtements de rechange pendant la désinfection des habits.

\section{Protection du personnel chargé de l'épouillage.}

Une protection absolue contre l'infection du typhus exanthématique et la fièvre récurrente n'est réalisable que par une hygiène corporelle constante ou par une action antérieure immunisante. Il est impossible que le personnel qui examine et épouille les malades et leurs effets soit totalement à l'abri de l'infection. Les épidémies de typhus exanthématique et de fièvre récurrente ne surviennent que dans une population largement infestée de poux. Si l'on vit et travaille parmi les malades, il est évident qu'à un moment donné la transmission de poux devient inévitable.

Une certaine protection peut être obtenue en portant des culottes d'équitation, des bottes, des habits et sous-vêtements fermant bien au cou et aux poignets, et surtout en changeant d'habit le soir, après le travail, afin d'épouiller préventivement les vêtements qui pourraient être infestés.

Pour les médecins, les infirmières et le personnel de désinfection, la vaccination active contre le typhus exanthématique est sérieusement à envisager, à côté d'une propreté méticuleuse du corps et des vêtements. En revanche, il n'existe pas encore de vaccin contre la fièvre récurrente épidémique. L'immunisation pratiquée sur une grande échelle, pendant une épidémie de typhus exanthématique, n'est pas réalisable à cause du prix de revient élevé et de la petite quantité de vaccin que les méthodes actuelles permettent d'obtenir. En outre, pour immuniser à l'aide de vaccin tué, on a besoin de 3 injections tous les 5-7 jours. Quant à l'efficacité de la vaccination à l'aide de virus vivant atténué, comme elle fut pratiquée en grand par les Français en Afrique septentrionale, on ne peut pas se prononcer d'une manière certaine. Pour cette raison, je ne peux pas recommander cette méthode. 


\section{Protection des populations civiles}

Les masques à gaz sont indispensables pour tout le personnel de désinsectisation, car, lors de la manipulation des vêtements, les excréments des poux infectés sont réduits en poussière et deviennent ainsi très dangereux, l'infection se propageant, non seulement par la morsure du pou infecté ou par le grattage ou l'écrasement du pou sur la peau, mais encore par l'inhalation des excréments pulvérisés et par leur pénétration dans les yeux.

\section{PROTECTION DES POPULATIONS CIVILES}

\section{Allemagne}

\section{Mesures de défense passive}

La Sirène, organe officiel de la "Ligue de défense aérienne du Reich ", a publié dans son numéro de décembre, un aperçu intéressant sur la protection aérienne civile allemande durant l'année I94I.

A la base de cette action de secours figure le décret du Fuhrer, sur l'intensification des mesures à prendre pour protéger les populations civiles ("Aktivierungserlass»), qui a prescrit et donné pratiquement une extension considérable aux différents services de la défense passive du Reich. En particulier, le sujet important des gardiens de la défense aérienne passive a été reconsidéré pour tout ce qui a trait à leur recrutement et à leur activité qui, elle, doit satisfaire aux exigences les plus sévères. Actuellement, un grand nombre d'officiers, de hauts fonctionnaires, et d'hommes du parti national-socialiste font leur temps de service militaire comme gardiens de la D.A.P. et fournissent un travail très efficace grâce à leur autorité et à leurs compétences. D'autre part, un grand nombre de femmes, employées depuis quelques années comme "agents" de la défense passive, ont pu être relevées de leurs fonctions afin qu'elles ne soient pas exposées plus longtemps, de manière directe, aux dangers des attaques aériennes. Ce renforcement systématique de la garde des immeubles a donné jusqu'ici d'excellents résultats. D'autre part, la livraison de couchettes par le Gouvernement du Reich, pour les abris individuels ou collectifs, est aussi à men- 